\title{
An MPEG-4 Performance Study for non-SIMD, General Purpose Architectures
}

\author{
Sally A. McKee \\ Electrical and Computer Engineering \\ Cornell University \\ Ithaca, NY 14853
}

\author{
Zhen Fang \\ School of Computing \\ University of Utah \\ Salt Lake City, UT 84112
}

\author{
Mateo Valero \\ Departament d'Arquitectura de Computadors \\ Universitat Politècnica de Catalunya \\ E-08071 Barcelona, Spain
}

\begin{abstract}
MPEG-4 is an important international standard with wide applicability. This paper focuses on MPEG-4's main profile, video, whose approach allows more effciency in coding and more flexibility in managing het. erogeneous media objects than previous MPEG standards. This study presents evidence to support the assertion that for non-SIMD architectures and computational models, most memory-system optimizations will have little effect on MPEG-4 performance. This paper makes two contributions. First, it serves as an independent confirmation that for current, general-purpose architectures, MPEG-4 video is computation bound (just like most other media processing applications). Second, our findings should prove useful to other researchers and practitioners considering how to (or how not to) optimize MPEG-4 performance.
\end{abstract}

\section{Introduction}

ISO/IEC 14496 [9], generally known as MPEG$4[14,10]$, is an important international standard whose immediate applications range from digital television and internet streaming video to mobile multimedia and games. MPEG-4 defines four main layers or profiles: control, still image, video and audio. We focus on the main profile, video, whose approach allows more efficiency in coding and more flexibility in managing heterogeneous media objects than its predecessors.

Two features distinguish MPEG-4 from MPEG-1 and MPEG-2: interactivity and streaming. Scene construction can be overlapped with image download. The decomposition of media data into objects provides two main advantages. First, it maximizes the efficiency of compression and encryption by introducing the potential to extract uncorrelated media content from the same source (and to apply the best media processing strat- egy for different types of content). Second, it allows a single protocol to manage a broad range of heterogeneous media content. Uncorrelated objects are coded, encrypted, and transmitted separately. At the reception site, powerful transformations (including zooming, rotation, or translation of image objects) may be performed over each object to recompose the audiovisual scene.

This new media standard thus enables interesting new functionalities, but poses difficult challenges with respect to computational capabilities. The "conventional wisdom" holds that the large working sets of streaming multimedia applications do not make effective use of large caches $[6,18,19]$, which leaves such applications hungry for bus bandwidth. ${ }^{1}$ Kuroda and Nishitani [15] show that the large data volumes for MPEG-2 motion compensation cause cache misses and main memory bandwidth to be severe performance problems. They hold that the memory/bus bottleneck is a prohibitive obstacle for MPEG on general-purpose architectures. Note that there is little difference between MPEG-2 and MPEG-4 in motion compensation. It follows that traditional cache- and bus-based memory hierarchies must be insufficient for this new application domain.

Many have found media processing to be computationally intensive on modern platforms $[24,12,18$, 3 , 11], but most of this work focuses on MPEG-1 or MPEG-2 kernels. With its new, real-time streaming feature, MPEG-4 poses a potential nightmare for a traditional memory hierarchy with shared memory buses. Media ISA extensions (e.g., Intel's( $\mathbb{R}$ MMX and SSE, AMD's( 3 DNOW!, Sun's VIS ${ }^{\mathrm{TM}}$, and Motorola's AltiVec ${ }^{\mathrm{TM}}$ ) can improve media-processing performance. ${ }^{2}$ SIMD-tuned compilers to exploit these exist, but getting the best performance often requires that programmers insert calls to assembly code libraries, use

\footnotetext{
'The literature can be confusing on these points - authors who recognize the high data locality of multimedia kemels still assume that cache and bandwidth utilization will be problematic.

${ }^{2}$ Jennings and Conte provide a succinct overview of many of these [11].
} 
special data structures, or hand-instrument source or assembly code. In fact, the "lagging compilers" lead Conte et al. [2] to assert that adding multimedia extensions to a general-purpose processor may not be an appropriate solution for multimedia workloads, while others maintain that these ISA extensions will make general-purpose processors more efficient and affordable than custom media accelerators [6]. Appropriate or not, most commercial multimedia applications now run on platforms that support these extensions, and thus have been be tuned to take advantage of them. These applications can also be of interest in other contexts [26]. For instance, we study how MPEG-4's real-time streaming feature affects memory performance on high-performance graphics machines. The original motivation was to assess the opportunity for improving performance via memorysystem optimizations, but we find that the code behaves contrary to our expectations.

This paper is intended to help overcome certain misconceptions about MPEG-4 behavior with respect to main-memory-utilization (particularly for highperformance platforms lacking SIMD ISA extensions). While perhaps-not prevalent in the literature, we: have often heard these misconceptions often in informal discussions on MPEG-4, and had held them ourselves prior to conducting this study.

We find the following assumptions to be false:

- MPEG-4 is a memory-streaming application.

- MPEG-4's performance is limited by busbandwidth.

- MPEG-4's performance is limited by latency.

- MPEG-4's performance is adversely affected by larger image sizes.

- MPEG-4's performance is adversely affected by a greater number of images or layers.

We target the Computer Architecture research community to help others avoid proposing architectural enhancements not needed for multimedia. We focus on a single profile, and we study a single implementation of MPEG-4. We do not experiment with MPEG-4 audio here, but our experience suggests it will present no problem to cache performance: MP3 audio applications [20], GSM long-term frequency vocoders [1], and similar codes are cache-friendly, since they also work at the frame level (one dimension, in this case), and since filtering and convolution operations (common in audio) have high temporal and spatial data locality.

This work is not intended to be exhaustive, but we believe our preliminary evidence sufficiently compelling to support the assertion that for non-SIMD architectures and computational models, most memory-systern optimizations will fail to deliver significant improvement on
MPEG-4 performance. The contributions of this paper are thus twofold. First, it serves as independent confirmation that for current, general-purpose architectures, MPEG-4 video is computation bound, just like most other media-processing applications. MPEG-4's streaming functionality does not affect this property of multimedia codes. Second, our findings should prove useful to other researchers and practitioners who may consider how to (or how not to) optimize MPEG-4 performance.

\section{Background}

This section first provides a brief tutorial on the video profile of the MPEG-4 standard, and then surveys the conclusions drawn from related MPEG performance characterization and architectural studies.

\subsection{Overview}

We provide an introduction to the MPEG-4 visual profile, but the interested reader may find much more detailed information elsewhere [10]. For reference, Jennings and Conte [11] provide a concise overview of MPEG-2 operation. The MPEG-4 standard's objectbased approach hinges on the central concept of the $v i$ sual object, or VO. A visual object corresponds to a particular 2-D object in the scene, and is characterized by temporal and spatial information in the form of shape, motion, and texture. VOs are sampled in time, and each can be encoded in scalable (multi-layer) or non-scalable (single layer) form.

Each time sample of a video object constitutes a video object plane, or VOP, containing motion parameters, shape information, and texture data. VOPs are encoded using $16 \times 16$ or $8 \times 8$ macroblocks, where a macroblock contains a section of the luminance component and the spatially subsampled chrominance components. Texture is coded separately by a discrete cosine transform (DCT) scheme. Arbitrary shapes are coded using a context-based arithmetic encoding scheme and are compressed via a bitmap-based method. Motion estimation and compensation are used in video compression to exploit temporal redundancies between frames. Whereas MPEG-1 and MPEG-2's motion estimation uses block-based techniques, MPEG-4's motion estimation has been adapted to VOPs.

Figure 1 illustrates how motion estimation is used in coding visual object planes, with arrows indicating interframe dependences. An Intra VOP, or $I-V O P$, is encoded independently and contains a complete image that is compressed for spatial redundancy only. A forward Predicted VOP, or $P-V O P$, is built from the nearest previously coded VOP. A Bidirectional VOP, or $B-V O P$, is interpolated based on I-VOPs and P-VOPs. Pulling 


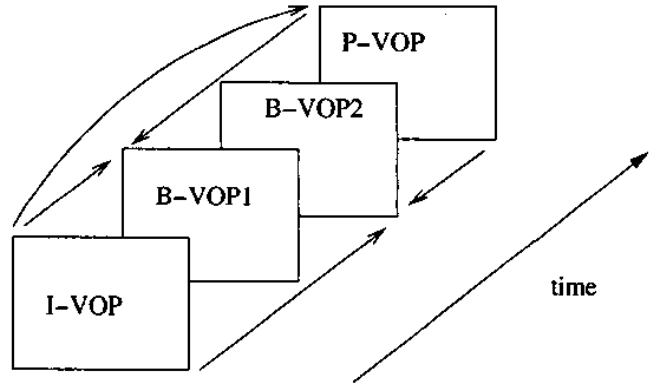

Figure 1. Three Modes of VOP Coding in MPEG-4

data from both past and future frames offers excellent opportunities for compression, but introduces data dependences. The decoder reads a stream of bits looking for the unique bit patterns called startcodes that mark the divisions between different sections of data in the hierarchical structure described above. The decoder must follow the operation-order dependences decided by the encoder. The VOPs are thus processed in the non-temporal order (I-VOP, P-VOP, B-VOP1, B-VOP $2, \ldots$ ). In other words, when the display order is I, B1, B2, P, the encoding and decoding orders are both $\mathrm{I}, \mathrm{P}, \mathrm{B} 1, \mathrm{~B} 2$. This out-of-order decoding (with respect to temporality) increases the performance and storage requirements for real-time playback.

\subsection{Related Work}

Since we approach MPEG-4 from a memoryperformance perspective, we briefly survey related work in the same vein. Space limitations prevent us from providing a comprehensive survey of background work rather, we strive to convey the flavor of prior work examining media application performance and contributing to our former misconceptions. We wish to stress that the claims and findings of those cited here are not necessarily incorrect in other contexts (e.g., with older workloads and processor models, or with SIMD ISA extensions), but instead that it may be inappropriate to infer that these conclusions also apply to MPEG-4 on generalpurpose platforms.

Many researchers, the authors included, have claimed that streaming access patterns and large working sets in multimedia applications make ineffective use of caches $[6,18,19,25]$.

Prefetching is one method commonly advocated to attack the DRAM latency problem for media applications $[16,6,17,26]$. For example, in their IA32 SIMD Streaming Extensions, Raman [17] et al. maintain that software prefetching will be important in supporting future streaming media applications. The rationale behind this is that these applications, especially MPEG-
4, are memory-latency bound. Zucker et al. [26] make this assumption in their trace-based study of hardware and software prefetching for MPEG benchmarks on the PA-RISC architecture. We assume their results are for traces generated by MPEG-2 applications, but this is not explicitly stated. This need for prefetching contradicts our findings for MPEG-4. In other contexts, e.g., systems that exploit SIMD multimedia instruction sets, prefetching can be profitable $[19,21]$. Extrapolating to assume prefetching works well on general-purpose computers lacking such ISA extensions or on applications not linked with SIMD libraries is potentially erroneous, as we show below.

Bus bandwidth is almost unanimously considered a bottleneck for multimedia $[6,13]$. The general claim is that the demand for continuous media data in applications like MPEG-4 makes their performance severely limited by bus bandwidth (internally and externally). We find that for our experiments on readily available systems, only a small fraction of the available bus bandwidth is needed. Ironically, this is due to good cache performance. Zucker et al. [26] assume bus bandwidth will not be a problem in future processors, but they do so based on an economic argument, not based on an analysis of cache performance.

Another closely related, popular misconception is that higher resolution in video will significantly increase memory usage, thus exacerbating the memorywall problem. For example, Ranganathan [18] et al.. conclude that a $1024 \times 1024$ image requires a factor of $12 \times$ increase in L2 cache size over that needed for a $352 \times 240$ image. We find that even at extremely high resolution images $(2048 \times 1024$ pixels, for example), the cache performance of MPEG-4 video remains at approximately the same level. Our findings agree with the later MPEG-2 working set studies of Hughes et al.. [8], in which they scale image input sizes as part of their research on the variability of multimedia application performance. This independence from image size is counterintuitive.

\section{Experimental Results}

In this section, we first describe our experimental setup. Then we address each of the misconceptions about performance that we initially held, and explain how our experience refutes them.

\subsection{Experimental Methodology}

We experiment on three machines: an SGI O2 (MIPS R12000 with 1MB L2 cache), an SGI Onyx VTX (MIPS R10000 with 2MB L2 cache) and an SGI Onyx2 InfiniteReality (MIPS R12000 with 8MB L2 cache). Ta- 


\begin{tabular}{|l|c|}
\hline Component & Features \\
\hline L1 data cache & $\begin{array}{c}\text { 32KB, 2-way set associative, } \\
\text { 32 bytes/line, LAU, WB }\end{array}$ \\
\hline L2 unified cache & $\begin{array}{c}\text { 2-way pseudo set associative, } \\
\text { 128 bytes/ine, LRU, WB } \\
\text { (size varies for each machine) }\end{array}$ \\
\hline system bus & 64 bits, 133 MHz, split transaction \\
\hline main memory & $\begin{array}{c}\text { 4-way interleaved SDRAM } \\
680 \mathrm{MB} / \mathrm{s} \text { sustained, } 800 \mathrm{MB} / \mathrm{s} \text { peal: }\end{array}$ \\
\hline operating system & IRIX64 v6.5 \\
\hline
\end{tabular}

Table 1. Common Platform Highlights

ble 1 highlights the relevant, common features of these three systems. The Irix kernel implements 32 virtual performance counters via multiplexing two actual hardware counters. We use the SGI SpeedShop [23] performance analysis package and the Irix Perfex [22] profiling tool library to access these counters. We use the publicly available MPEG-4 visual encoder and decoder from the ISO reference software developed by the European Union ACTS project MoMuSys (Mobile Multimedia Systems) [7]. We compile with the MIPS CC compiler at optimization level -03 .

Our experiments manipulate a 30 -frame video at two resolutions: the $720 \times 576$ used for PAL [5], and a $1024 \times 768$ size that exceeds NTSC but is less than HDTV [5]. Pixel depth is eight bits. The frame rate is $30 \mathrm{~Hz}$, as in HDTV (note that PAL uses a $25 \mathrm{~Hz}$ rate), and the target bitrate is 38400 .

Table 2 and Table 3 summarize our MPEG-4 visual encoding and decoding experiments, respectively. The numbers for instruction cache and TLB misses are negligible, and are omitted. Cache line reuse is the mean number of times a cache line is used after being loaded and before being evicted. For example, LIC line reuse is the graduated loads plus graduated stores, minus L1 data cache misses, all divided by $\mathrm{L} 1$ data cache misses. Likewise, $L 2 C$ line reuse is $\mathrm{L} 1$ data cache misses minus L2 data misses, all divided by L2 data misses. DRAM time refers to the cycles during which the processor is stalled due to secondary data cache misses; this is the latency that out-of-order execution hardware and compilation techniques fail to hide. L2-DRAM $b / w$ is the amount of data moved between the secondary cache and main memory, divided by the total program execution time. The amount of data moved is calculated as the sum of the L2 cache misses multiplied by the L 2 cache line size, plus the number of bytes written back from $\mathrm{L} 2$. $L I \cdot L 2 b / w$ is similar. Prefetch $L I C$ miss refers to the proportion of prefetch instructions that do not become nops. A high prefetch miss rate (near one) is desirable, since prefetch hits waste instruction bandwidth and decoding resources. The MIPS R10000 cannot track the number of prefetches that hit in L1 cache; this statistic is only available on our MIPS R12000-based machines.

\subsection{Fallacies and Paradoxes}

We now examine in turn each of the popular assumptions described in Section 1.

Fallacy: MPEG-4 Exhibits Streaming References. Primary cache performance is nearly optimal across all hardware configurations and input sizes. Even at 1024 $\times 768$ pixels/frame, the $\mathrm{L} 1$ data cache hit rate is up to $99.91 \%$. Of the many data accesses that constitute the load streams and store streams dispatched by the processor, only $0.1 \%$ and $0.4 \%$ go beyond $\mathrm{Ll}$ cache for encoding and decoding, respectively. These high hit ratios make L1 data misses account for less than $0.50 \%$ and $1.76 \%$ of execution time in encoding and decoding, respectively. On average, each $\mathrm{L} 1$ cache line is reused about 1000 times before eviction in encoding, and more than 200 times in decoding. The intuition that streaming MPEG-4 is a poor match for small caches is therefore false in this context: the data references in "streaming MPEG-4" do not really stream.

This phenomenon occurs because, in spite of the streaming nature of the kernels used, their composition into multimedia programs generates locality in two ways: (a) streams have high degrees of data overlap, and (b) different stages of the application's "pipeline" process the same data resident in L1 cache. Furthermore, the MPEG-4 protocol itself dictates that data be organized in chunks (e.g., $16 \times 16$ elements in motion estimation or $8 \times 8$ in discrete cosine transform).

Consider the encoder's motion estimation (responsible for the majority of the program execution time). Motion estimation detects movement of objects along different video frames, searching for an image block best matching a reference block. The "resemblance" criterion is the minimum sum of absolute differences(SAD) between pixels of the two blocks. From the computation kernel's perspective, processing data streams across different blocks exhibits little data locality. MPEG-4 performs this search sequentially over restricted windows inside the image, with an offset between searches of just one pixel. The overlap among streams for searching an image subset yields high locality both in the $x$ axis, due to the data layout in memory, and in the $y$ axis, due to the restricted size of the window. Simply put, the protocoldictated blocking structure naturally creates locality.

Fallacy: MPEG-4 is Bound by DRAM Latency. Memory requirements for MPEG-4 video processing are indeed large. This fact gives rise to the common assumption that memory latency is critical to MPEG-4 performance, especially since much MPEG-4 data is timesensitive. In our experiments, over $99.5 \%$ of the data references hit the primary cache, and even a small $1 \mathrm{MB}$ secondary cache catches more than $60 \%$ of the rest. Very few references reach main memory. Out-of-order issue 


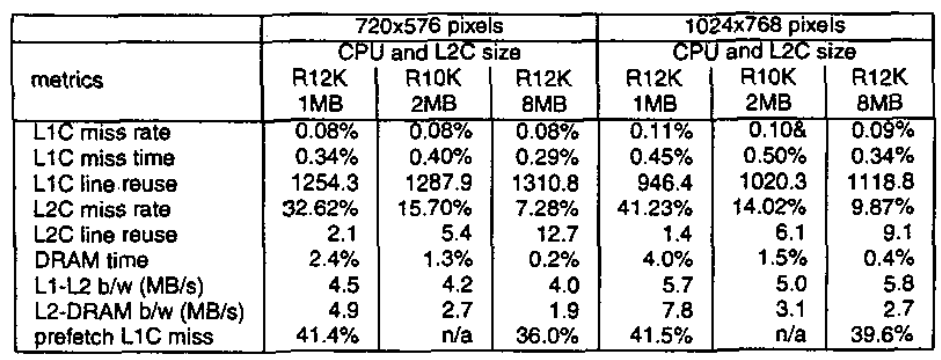

Table 2. Video Encoding: One Visual Object, One Layer

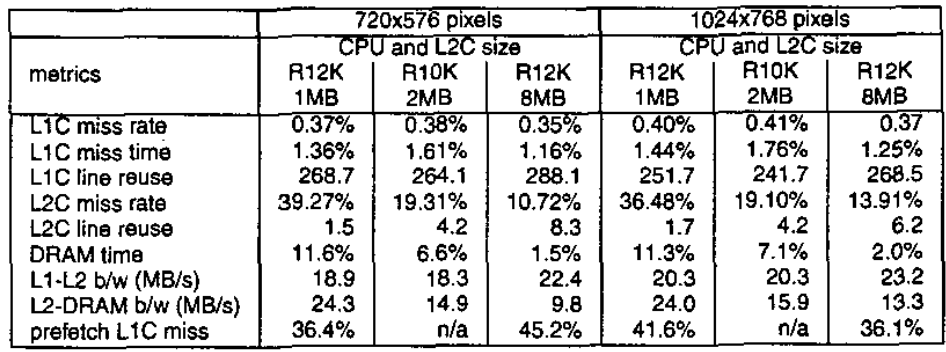

Table 3. Video Decoding: One Visual Object, One Layer

and the MIPS optimizing compiler hide another portion of the latency of these main-memory references. For encoding on a system with a large L2 cache, the processor stalls as low as $0.2 \%$ of the time for medium-sized 720 $\times 576$ frames. Even for a small L2C and large $1024 \times$ 768 frames, the processor stalls only about $4.0 \%$ of the time. Decoding spends slightly more time on processor stalls while waiting for DRAM accesses. In the worst case, we observe a processor stall time of no more than $12 \%$. In spite of initial claims that multimedia codes do not use caches well, Ranganathan et al. [18, 19] observe similarly small percentages of memory stall time (less than 10\%) for their studies of MPEG-2 and other multimedia codecs.

With compiler-generated software prefetching, the number of executed prefetches is around $1 / 7000$ the number of graduated loads in encoding and $1 / 1000$ in decoding (not shown in the tables). Even for this conservative use of prefetching, over half of the prefetches hit the primary cache, and thus constitute a waste of system resources. Prefetching is therefore unlikely to improve MPEG 4 performance on the systems we study.

Fallacy: MPEG-4 is Hungry for Bus Bandwidth. In terms of sustained bandwidth, traffic is less than $2 \%$ between the $L 1$ and $L 2$ caches, and less than $4 \%$ between L2 cache and main memory. Again, this comes from high reuse and high primary cache hit rates. Given the high L1C hit rate, bandwidth between the ALUs and the primary cache might be a limiting factor, but the R 10000 and R 12000 counters prevent exploration of this

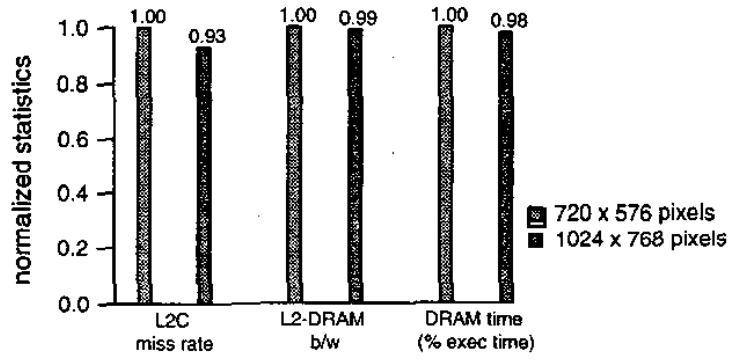

Figure 2. Memory Statistics for Growing Image Size (Decoding, 1MB L2C)

conjecture. Simulation studies [4] indicate that a nonSIMD/non-vector MPEG-4 code with a dual-ported LIC is not limited by bandwidth constraints, while a vector version is, even with a four-ported cache.

Fallacy: MPEG-4 Memory Performance Degrades with Growing Image Size. At first glance, this extrapolated assumption from image processing experiences seems plausible, even likely. Nonetheless, our results suggest otherwise. Despite the fact that memory requirements grow about linearly with respect to image size, performance remains almost the same when the image size is almost doubled (from $720 \times 576$ to $1024 \times 768$, a factor of 1.9 ). Even with extremely large frames (2048 $\times 1024$ pixels) we see equally good memory performance (data not shown here). The blocking nature of the algorithms makes the image size largely 


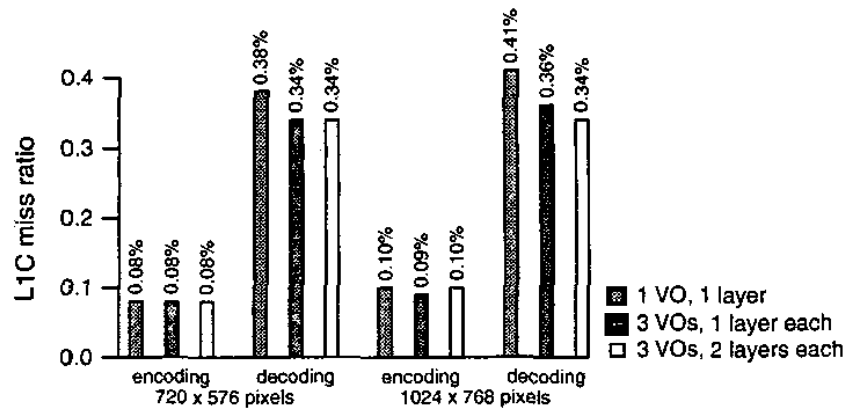

Figure 3. L1C Miss Rates for Varying Numbers of Objects and Layers

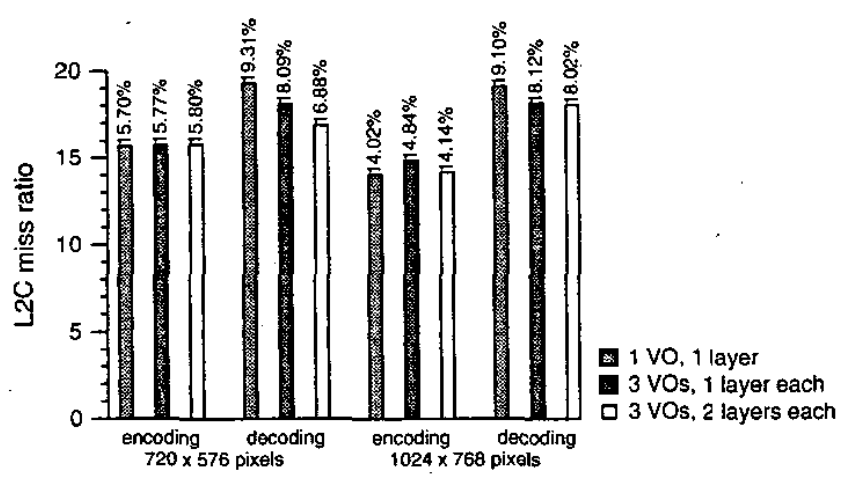

Figure 4. L2C Miss Rates for Varying Numbers of Objects and Layers

irrelevant. Interestingly, in several cases memory performance actually improves as the image size increases. For example, for decoding on a system with a 1MB L2, as the image size grows, the L2 miss rate, memory bandwidth consumption and DRAM stall time all decrease, as shown in Figure 2. Counterintuitively, cache performance of MPEG-4 video proves to be independent of frame size.

Fallacy: MPEG-4 Memory Performance Degrades as the Number of Visual Objects and Layers Grows. Thus far, we have concentrated on memory statistics for a single visual object with one layer. Increasing the number of visual objects (VOs) and visual object layers (VOLs) increases memory requirements accordingly. For example, on a workload of ( $1 \mathrm{VO}, 1$ VOL, $1024 \times 768$ ), encoding uses about $120 \mathrm{MB}$.of stable, resident memory. At ( 3 VOs, 2 VOLs, $1024 \times 768$ ) encoding requires $400 \mathrm{MB}$. Intuition might suggest that cache performance would degrade as multiple VOs and VOLs compete for system resources. Surprisingly, we find this not to be the case. Table 4 and Table 5 contain statistics for processing three visual objects. Each of the three objects is encoded and decoded for the same configuration as in the single object experiments, with the single-object input becoming a subset of the multipleobject input. Table 6 and Table 7 present the numbers for three visual objects with two visual object layers each. Table 4, Table 5, Table 6, and Table 7 with Table 2 and Table 3 show that cache performance does not change noticeably as the number of VOs and VOLs increases. Figure 3 and Figure 4 visually depict part of this comparison, showing $\mathrm{L} 1 \mathrm{C}$ and $\mathrm{L} 2 \mathrm{C}$ data miss rates on an $\mathrm{R} 10 \mathrm{~K}$ machine with an L2 cache of $2 \mathrm{MB}$ (note the different orders of magnitude in the $y$ axis scales). More interesting is the change in performance for the decoding process as the number of VOs increases. Cache performance actually improves as we go from single object/single layer (Table 3), to multiple objects/single layer (Table 5), and up to multiple objects/muitiple layers (Table 7). Consider the $1024 \times 768$ case. The $\mathrm{L} 1$ cache miss rates drop from $0.41 \%$ to $0.36 \%$ and $0.34 \%$. L2 cache miss rates drop from $19.10 \%$ to $18.12 \%$ and $18.02 \%$. And as a result, DRAM stall time drops from $7.1 \%$ to $5.9 \%$ and $5.6 \%$. A similar trend occurs for the $720 \times 576$ case. This paradoxical behavior of "improving under pressure" reinforces our conclusions about MPEG-4 performance on the platforms we study.

\subsection{Burstiness}

To detect the inherent burstiness of MPEG-4 memory traffic, we instrument two of the most important functions - VopCode() in the encoder and DecodeVopCombMotionShapeTexture() in the decoder - by wrapping them in performance counter operations. Specifically, we want to determine if these two functions exhibit burstier memory behavior compared to the rest of MPEG-4 video. VopCode() performs shape, texture and motion coding of the input visual object plane, generating an encoded VOP. Motion estimation is the most time-consuming function in the VOP encoding process. DecodeVopCombMotionShapeTexture() is the reverse of VopCode(), decoding a VOP from the input bitstream. $2 \mathrm{D}$ DCT is the primary technique used in the decoding process. In the literature, motion estimation and DCT are the most frequently used examples for MPEG4 memory optimizations. We do not apply instrumentation to the macroblock level of motion estimation or DCT, since at such fine granularity the inserted system calls would affect the execution noticeably and the accumulated error would have been unacceptable.

In Table 8, the functions VopCode() and DecodeVopCombMotionShapeTexture() are renamed VopEncode and VopDecode, respectively. Data are collected on a (R12K, $8 \mathrm{MB} \mathrm{L2C})$ machine. 


\begin{tabular}{|c|c|c|c|c|c|c|}
\hline \multirow[b]{3}{*}{ metrics } & \multicolumn{3}{|c|}{$720 \times 576$ pixels } & \multicolumn{3}{|c|}{$1024 \times 768$ pixels } \\
\hline & \multicolumn{3}{|c|}{ CPU and L2C size } & \multicolumn{3}{|c|}{ CPU and $2 \mathrm{C}$ size } \\
\hline & $\begin{array}{l}\mathrm{R} 12 \mathrm{~K} \\
1 \mathrm{MB}\end{array}$ & $\begin{array}{l}\text { R1OK } \\
2 \mathrm{MB}\end{array}$ & $\begin{array}{l}\text { R12K } \\
8 \mathrm{MB}\end{array}$ & $\begin{array}{l}\mathrm{R} 12 \mathrm{~K} \\
1 \mathrm{MB}\end{array}$ & $\begin{array}{l}\text { R1OK } \\
2 \mathrm{MB}\end{array}$ & $\begin{array}{l}R 12 \mathrm{~K} \\
8 \mathrm{MB}\end{array}$ \\
\hline Lic miss rate & $0.09 \%$ & $.0 .08 \%$ & $0.08 \%$ & $0.10 \%$ & 0.098 & $0.09 \%$ \\
\hline L1C miss time & $0.35 \%$ & $0.40 \%$ & $0.27 \%$ & $0.41 \%$ & $0.48 \%$ & $0.32 \%$ \\
\hline L1C line reuse & 1172.9 & 1235.6 & 1276.2 & 999.3 & 1060.6 & 1091.1 \\
\hline L2C miss rate & $32.24 \%$ & $15.77 \%$ & $10.04 \%$ & $33.02 \%$ & $14.84 \%$ & $10.97 \%$ \\
\hline L2C line reuse & 2.1 & 5.3 & 9.0 & 2.0 & 5.7 & 8.1 \\
\hline DRAM time & $2.4 \%$ & $1.4 \%$ & $0.3 \%$ & $2.9 \%$ & $1.5 \%$ & $0.4 \%$ \\
\hline L1-L2 b/w (MB/s) & 4.5 & 4.3 & 4.7 & 5.2 & 4.9 & 5.4 \\
\hline L2-DRAM b/w (MB/s) & 4.9 & 2.8 & 2.0 & 5.8 & 3.1 & 2.6 \\
\hline prefetch $L 1 C$ miss & $39.6 \%$ & $n / a$ & $41.7 \%$ & $42.5 \%$ & $n / a$ & $42.5 \%$ \\
\hline
\end{tabular}

Table 4. Video Encoding: Three Visual Objects, One Layer Each

\begin{tabular}{|c|c|c|c|c|c|c|}
\hline \multirow[b]{3}{*}{ metrics } & \multicolumn{3}{|c|}{$720 \times 576$ pixels } & \multicolumn{3}{|c|}{$1024 \times 768$ pixels } \\
\hline & \multicolumn{3}{|c|}{ CPU and $L 2 C$ size } & \multicolumn{3}{|c|}{ CPU and $12 \mathrm{C}$ size } \\
\hline & $\begin{array}{c}\text { R12K } \\
1 \mathrm{MB}\end{array}$ & $\begin{array}{c}\text { P10K } \\
2 \mathrm{MB}\end{array}$ & $\begin{array}{c}\text { R12K } \\
8 \mathrm{MB}\end{array}$ & $\begin{array}{c}\text { R12K } \\
\text { 1MB }\end{array}$ & $\begin{array}{c}\text { R1OK } \\
2 \mathrm{MB}\end{array}$ & $\begin{array}{c}\mathrm{A} 12 \mathrm{~K} \\
8 \mathrm{MB}\end{array}$ \\
\hline L1C miss rate & $0.31 \%$ & $0.34 \%$ & $0.28 \%$ & $0.33 \%$ & $0.36 \%$ & 0.30 \\
\hline L1C miss time & $1.20 \%$ & $1.46 \%$ & $0.96 \%$ & $1.27 \%$ & $1.52 \%$ & $1.06 \%$ \\
\hline L1C line reuse & 318.6 & 291.5 & 358.6 & 299.3 & 280.3 & 327.9 \\
\hline L2C miss rate & $36.56 \%$ & $18.09 \%$ & $12.41 \%$ & $35.22 \%$ & $18.12 \%$ & $14.92 \%$ \\
\hline $\mathrm{L} 2 \mathrm{C}$ line reuse & 1.7 & 4.5 & 7.1 & 1.8 & 4.5 & \\
\hline DRAM time & $9.5 \%$ & $5.6 \%$ & $1.4 \%$ & $9.7 \%$ & $5.9 \%$ & $1.9 \%$ \\
\hline $\mathrm{L} 1 . \mathrm{L} 2 \mathrm{~b} / \mathrm{w}(\mathrm{MB} / \mathrm{s})$ & 16.8 & 16.7 & 17.8 & 17.9 & 17.3 & 19.7 \\
\hline L2-DRAM b/w (MB/s) & 20.2 & 12.3 & 9.5 & 20.6 & 13.0 & 12.0 \\
\hline prefetch $L 1 C$ miss & $44.4 \%$ & $\mathrm{n} / \mathrm{a}$ & $40.3 \%$ & $41.2 \%$ & $n / a$ & $41.5 \%$ \\
\hline
\end{tabular}

Table 5. Video Decoding: Three Visual Objects, One Layer Each

\begin{tabular}{|l|r|r|r|r|r|r|}
\hline & \multicolumn{3}{|c|}{$720 \times 576$ pixels } & \multicolumn{3}{c|}{$1024 \times 768$ pixels } \\
\hline \multirow{2}{*}{ metrics } & \multicolumn{3}{|c|}{ CPU and L2C size } & \multicolumn{3}{c|}{ CPU and L2C size } \\
& R12K & R10K & R12K & R12K & R10K & R12K \\
& $1 \mathrm{MB}$ & $2 \mathrm{MB}$ & $8 \mathrm{MB}$ & \multicolumn{1}{c|}{$1 \mathrm{MB}$} & \multicolumn{1}{c|}{ 2MB } & \multicolumn{1}{c|}{$8 \mathrm{MB}$} \\
\hline L1C miss rate & $0.08 \%$ & $0.08 \%$ & $0.08 \%$ & $0.11 \%$ & 0.108 & $0.10 \%$ \\
L1C miss time & $0.34 \%$ & $0.41 \%$ & $0.29 \%$ & $0.45 \%$ & $0.51 \%$ & $0.35 \%$ \\
L1C line reuse & 1179.1 & 1202.7 & 1249.4 & 910.5 & 966.9 & 1028.3 \\
L2C miss rate & $32.27 \%$ & $15.80 \%$ & $9.97 \%$ & $40.83 \%$ & $14.14 \%$ & $10.15 \%$ \\
L2C line reuse & 2.1 & 5.3 & 9.0 & 1.4 & 6.1 & 8.9 \\
DRAM time & $2.4 \%$ & $1.4 \%$ & $0.3 \%$ & $4.0 \%$ & $1.5 \%$ & $0.4 \%$ \\
L1-L2 b/w (MB/s) & 4.5 & 4.3 & 4.9 & 5.7 & 5.2 & 5.9 \\
L2-DRAM b/w (MB/s) & 4.9 & 2.8 & 2.1 & 7.8 & 3.2 & 2.6 \\
prefetch L1C miss & $41.7 \%$ & n/a & $43.8 \%$ & $43.6 \%$ & n/a & $40.6 \%$ \\
\hline
\end{tabular}

Table 6. Video Encoding: Three Visual Objects, Two Layers Each

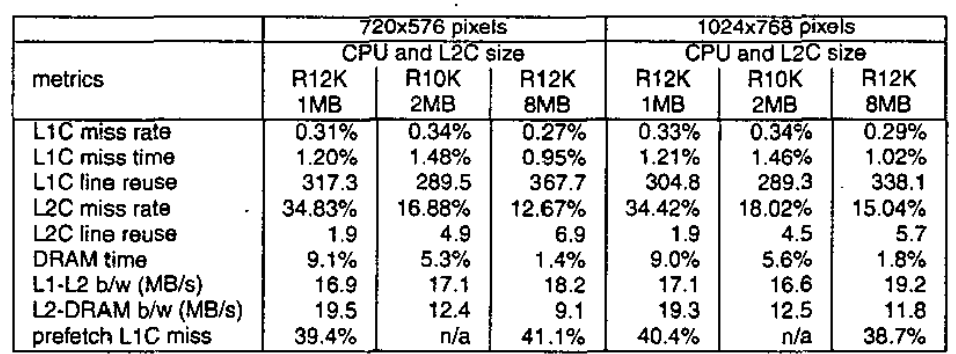

Table 7. Video Decoding: Three Visual Objects, Two Layers Each 


\begin{tabular}{|c|c|c|c|c|}
\hline & \multicolumn{2}{|c|}{ VopEncode } & \multicolumn{2}{|c|}{ VopDecode } \\
\hline metrics & $\begin{array}{l}3 \text { visual objects } \\
1 \text { layer each } \\
720 \times 576 \text { pixels }\end{array}$ & $\begin{array}{c}3 \text { visual objects } \\
2 \text { layers each } \\
1024 \times 768 \text { pixels }\end{array}$ & $\begin{array}{l}3 \text { visual objects } \\
1 \text { layer each } \\
720 \times 576 \text { pixels }\end{array}$ & $\begin{array}{c}3 \text { visual objects } \\
2 \text { layers each } \\
1024 \times 768 \text { pixels }\end{array}$ \\
\hline $\begin{array}{l}\text { L1C miss rate } \\
\text { L2C miss rate } \\
\text { L1-L2 } \mathrm{b} / \mathrm{w}(\mathrm{MB} / \mathrm{s}) \\
\mathrm{L} 2-\mathrm{DAAM} \mathrm{b} / \mathrm{w}(\mathrm{MB} / \mathrm{s})\end{array}$ & $\begin{array}{r}0.05 \%(0.08 \%) \\
3.09 \%(10.04 \%) \\
5.7(4.7) \\
0.9(2.0)\end{array}$ & $\begin{array}{r}0.05 \%(0.10 \%) \\
4.04 \%(10.15 \%) \\
5.6(5.9) \\
1.3(2.6)\end{array}$ & $\begin{array}{r}0.72 \%(0.28 \%) \\
9.80 \%(12.41 \%) \\
38.3(17.8) \\
5.9(9.5)\end{array}$ & $\begin{array}{r}0.73 \%(0.29 \%) \\
11.33 \%(15.04 \%) \\
38.1(19.2) \\
6.8(11.8)\end{array}$ \\
\hline
\end{tabular}

Tabie 8. Encoding and Decoding a VOP

For convenient comparison, we include in brackets the whole-program statistics taken from previous lables. Inspection reveals that the memory performance of these two functions is consistent with overall trends. The L2C miss rate and L2-DRAM traffic are both smaller than in the whole program. VopEncode sees better memory performance than overall encoding for all metrics shown in Table 8. For VopDecode, Ll cache references miss about twice as often as the whole-program average, increasing L1C-L2C traffic. Even with this degraded L1C performance, the L1 cache still captures over $99.2 \%$ of all accesses in the load streams and store streams dispatched by the processor. Improved L2C hit rate further filters the memory references, reducing traffic to DRAM. This test of burstiness for key encoding/decoding phases tells us that, although each of the kemels (e.g. SAD and DCT) references data in a streaming manner, at the VOP level the comprehensive effect of multiple streams is a working set that fits well into cache.

\section{Conclusions and Future Work}

We find that for the experiments we conduct, the MPEG-4 video profile has good memory performance: high primary cache hit ratios, high cache-line reuse, low main-memory stall times, and low bus-bandwidth requirements. Although we experiment with generalpurpose processors lacking MMX-like SIMD extensions, our experience has shown that even in the presence of these ISA extensions, the performance bottleneck is still the fetch/issue rate [3]. Only in the presence of longer vector SIMD instructions does L,1 bandwidth surpass fetch rate as a limiting performance factor. Given our experiences, we caution against generalizing results from older workloads, different processor/ISA models, and different domains (e.g., image processing) to modern multimedia workloads on generalpurpose platforms. On the other hand, care should be taken when generalizing observations made in this paper to other platforms or MPEG-4 implementations. Fortunately, the increasing availability and accessibility of hardware performance counters in such platforms makes it easier to examine application behavior to understand where the performance bottlenecks are (or are not).
This work represents our preliminary findings. While performance numbers from commercial hardware using a commercial compiler have the advantage of being more realistic than those generated via software simulation, the parameters of our experiments are necessarily rigid. In order to investigate how MPEG- 4 behaves with different architectural configurations, we are extending our experiments to a spectrum of representative platforms (including IA32, IA64, and Power4). Our intuition is that the memory performance of the MPEG-4 visual profile is unlikely to change qualitatively on any mainstream workstation with a conventional cache hierarchy. These studies will also incorporate the effect of SIMD ISA extensions, evaluating the appropriateness of adding such extensions to general-purpose processors. Previous work [4] finds that vector versions of MPEG4 become $\mathrm{Ll}$ cache bandwidth-limited. Investigating the behavior of a non-vectorized MPEG-4 that exploits multimedia ISA extensions on general-purpose machines is a next step, and the following one is gathering data for other MPEG-4 profiles. Finally, we will conduct simulation studies to determine at what ratio of processorto-memory speed and at what bandwidths among various levels of the memory hierarchy the performance of MPEG-4 does finally become memory limited.

\section{Acknowledgments}

We thank Jesus Corbal for comments on drafts of this paper.

\section{References}

[1] S. Bonnett. MP3 technical information. http://whww. iocon.com/das/technical shtm1, 2001.

[2] T. Conte, P. Dubey, M. Jennings, R. Lee, A. Peleg, S. Rathnam, M. Schlansker, P. Song, and A. Wolfe. Challenges to combining general-purpose and multimedia processors. IEEE Computer, 30(12):33-37, Dec. 1997.

[3] J. Corbal, R. Espasa, and M. Valero. DLP + TLP processors for the next generation of media workloads. In Proceedings of the Seventh Annual Symposium on High Performance Computer Architecture, pages 219-228, Jan. 2001. 
[4] J. Corbal, R. Espasa, and M. Valero. Three-dimensional memory vectorization for high bandwidth media memory systems. In Proceedings of IEEEJACM 35th International Symposium on Microarchitecture, pages 149-160, Nov. 2002.

[5] CVC Productions. World TV standards. http://avconvert. com/video/worlatelevision standards.html, 2001.

[6] K. Diefendorff and P. Dubey. How multimedia workloads will change processor design. IEEE Computer, 30(9):43-45, Sept. 1997.

[7] European Union ACTS Programme. Mobile Multimedia Systems Project (MoMuSys). http:/ihww.intowin.org/ACTS/RUS/PROJECTS/ac09B.htm.

[8] C. Hughes, P. Kaul, S. Adve, R. Jain, C. Park, and J. Srinivasan. Variability in the execution of multimedia applications and implications for architecture. In Pro. ceedings of the 28th Annual International Symposium on Computer Architecture, pages 254-265, June 2001.

[9] ISO/EC 14496-2. Coding of audio-visual objects:visual, final draft international standard, Oct. 1998.

[10] ISO/IEC Moving Picture Experts Group. The MPEG Webpage. ntep://mpeg.telecomitalialab.con/, 2002.

[11] M. Jennings and T. Conte. Subword extensions for video processing on mobile systems. IEEE Concurrency, 6(3):13-16, July-September 1998.

[12] B. Khailany, W. Dally, S. Rixner, U. Kapasi, P. Mattson, J. Namkoong, J. Owens, B. Towles, and A. C. hang. Imagine: Media processing with streams. IEEE Micm, 21(2):35-46, March/April 2001.

[13] J. Kneip, B. Schmale, and H. Moller. Applying and implementing the MPEG-4 multimedia standard. IEEE Micro, 19(6):64-74, Nov. 1999.

[14] R. Koenen. Mpeg-4: Multimedia for our time. IEEE Spectrum, 36(2):26-34, Feb. 1999.

[15] I. Kuroda and T. Nishitani. Multimedia processors. Proceedings of the IEEE, 86(6):1203-1221, June 1998.

[16] A. Prati. Exploring multimedia applications locality to improve cache per formance. In ACM Multimedia, pages 509-510, 2000

[17] S. Raman, V. Pentkovski, and J. Keshava. Implementing streaming SIMD extemsions on the Pentium III processor. IEEE Computer, 33(7):47-57, July 2000.

[18] P. Ranganathan, S. Adve, and N. Jouppi. Performance of image and video processing with general-purpose processors and media isa extensions. In Proceedings of the 26th Annual International Symposium on Computer Ar. chitecture, pages 124-135, June 1999.

[19] P. Ranganathan, S. Adve, and N. Jouppi. Reconfigurable caches and their application to media processing. In Proceedings of the 27th Annual International Symposium on Computer Architecture, pages 214-224, June 2000.

[20] J. Scourias. GSM. http://mww. shoshin. uwaterloo.ca/ jscouria/GS M/index.htmi 1999.
[21] J. Sebot and N. Drach. Memory bandwidth: The true bottleneck of simd multimedia performance on a superscalar processor. In Proceedings of the 2001 European Conference on Parallel Computing, pages 439-447, Aug. 2001.

[22] Silicon Graphics, Inc. Origin 2000 and Onyx 2 Performance Tuning and Optimization Guide (document number: 007-3430-003). http://techpubs.sgi.com , 2001.

[23] Silicon Graphics, Inc. SpeedShop User's Guide (document number: 007-3311-007). http://techpubs. sgi .com:80/1ibrary/tpl/cgi-b in/init.cgi, 2001.

[24] N. Slingerland and A. Smith. Cache performance for multimedia applications. In Proceedings of the $2001 \mathrm{In}$ ternational Conference on Supercomputing, pages 204217, June 2001.

[25] L. Zhang, Z. Fang, M. Parker, B. Mathew, L. Schaelicke, J. Carter, W. Hsieh, and S. McKee. The impulse memory controller. IEEE Transactions on Computers, 50(11):1117-1132, Nov. 2001.

[26] D. Zucker, R. Lee, and M. Flynn. Hardware and software prefetching techniques for MPEG benchmarks. IEEE Transactions on Circuits and Systems for Video Technology, 5(10):782 -796, August 2000. 